\title{
Universality and Beyond
}

\author{
D. R. Phillips ${ }^{1,2, a}$ \\ 1 Department of Physics and Astronomy, Ohio University, Athens, Ohio 45701, USA \\ 2 Helmholtz-Institut für Strahlen- und Kernphysik, Universität Bonn, D-53115, Bonn, Germany
}

\begin{abstract}
I discuss the impact of a finite effective range, $r$, on systems with a large two-body scattering length, a. In particular, I show how observables can be written as an expansion around the "universal", or large-scatteringlength limit. The parameter governing this expansion is the ratio $r / a$. In few-nucleon systems the ratio $r / a$ has a value of about $1 / 3$, and so such corrections are essential in producing good agreement between theory and data. Hence, I first show how these effects range play a key role in making the so-called "pionless" effective field theory a successful descriptor of low-energy processes in the NN system. I then move to the NNN system, and review predictions for the energy-dependence of observables there. However, the beautiful Efimov physics associated with the presence of a large scattering length is not fully revealed in the NNN system, precisely because $r / a$ corrections are large. I therefore turn to cold atomic gases and show that there are some important recent experiments where physics "beyond universality" affects the data. In the process I demonstrate that an additional piece of short-distance physics is necessary to renormalize scattering-length-dependent observables in the three-body system once corrections $\sim r$ are considered. Finally, I discuss recent initial efforts to compute $r / a$ corrections to the predictions of universality for the four-body system.
\end{abstract}

\section{Introduction}

In the context of few-body systems, "universality" refers to two-, three-, and four-body systems that have properties which are independent of the details of the underlying interaction. This universality emerges in systems with a two-body scattering length $a$ that is large compared to the range of the interaction $\ell$. Under such circumstances the details of the interaction play only a sub-leading role in determining the observable quantities. Under the rubric of "universality" we collect systems as diverse as cold atoms, the X(3872), halo nuclei, and few-nucleon systems. We see that their bound (and, in some cases, low-energy scattering) states share certain common features, even though the microscopic interactions are quite different in all cases. Table 1 collects these systems and showcases the hugely different length scales involved. What Table 1 makes clear, though, is that in all these systems $\ell /|a|$ is a small parameter.

This means that observables can be expanded in this small parameter. The leading-order prediction for observables is then given by the limit $\ell /|a|=0$. The predictions for this limit are discussed at length in the review of Ref. [1] as well as in the contribution of Braaten to this conference [2]. I will touch on this beautiful physics only briefly here, since my focus will be on the corrections that take us beyond the "universal" predictions. Those corrections are manifest in a variety of different observables, with the pertinent observables depending on the system under examination. In few-nucleon physics we are fortunate to

\footnotetext{
a e-mail: phillips@phy.ohiou.edu
}

Table 1. Some systems in which the presence of large scattering lengths results in observables displaying "universal" features. $\ell$ is the "characteristic length scale" of the interaction - the range of the force in the nuclear case, while $a$ is the scattering length, and here and throughout $a_{B}$ denotes the Bohr radius. Note that in this table the scattering length and range given for the NN system are taken from the ${ }^{3} S_{1}$ channel. The X(3872) will not be discussed further in this talk, but was covered in the contribution of Mehen to this meeting [3].

\begin{tabular}{|c|c|c|c|}
\hline System & $\ell$ & $\mathrm{a}$ & Observables \\
\hline $\begin{array}{l}\mathrm{Li} \text { atoms } \& \\
\text { Cs atoms }\end{array}$ & $\sim 100 a_{B}$ & Varies & Recombination \\
\hline X (3872) & $1.5 \mathrm{fm}$ & $\sim 10 \mathrm{fm}$ & Spectrum, decays \\
\hline Halo nuclei & $3 \mathrm{fm}$ & $\sim 10 \mathrm{fm}$ & $\begin{array}{l}\text { Spectra, scattering, } \\
\text { EM excitation }\end{array}$ \\
\hline $\mathrm{NN}, \mathrm{NNN}, \ldots$ & $1.7 \mathrm{fm}$ & $5.4 \mathrm{fm}$ & $\begin{array}{l}\text { Phase shifts, Spectra } \\
\text { EM properties }\end{array}$ \\
\hline
\end{tabular}

have a wealth of experimental data, including phase shifts and electromagnetic properties, all of which allow us to look at the effects of finite- $\ell$ on the low-energy properties of NN, NNN, and even NNNN systems.

These data are, however, restricted to a fixed value of $a$. In contrast, at low temperatures certain types of atoms (e.g. those listed in the first lines of Table 1) can be examined at different scattering lengths. The observables there are features in the recombination rates (and therefore losses) measured in trapped, cold gases of these species. Cold atomic gases therefore provide a complementary way to investigate corrections to universality. In few-nucleon systems we 
tend to examine observables as a function of energy, but at fixed $a$, while in atomic recombination we work at fixed (or, to be more precise, some average) energy but variable $a$. Both kinds of investigation provide useful ways to look for effects beyond universality.

Such effects are important because universality tells us how these diverse systems are similar, while the effects due to the finite range of the interaction illuminate their differences. Determining how to systematically incorporate those differences in the predictions for few-body-system observables is the subject of this talk. We will begin with the two-body system.

\section{The two-body system}

Since we are interested in systems for which the details of the interaction are not important we are free to work with interactions which are easy to calculate. In particular, we take the leading-order Lagrangian to be $[4,5]$ :

$$
\mathcal{L}=\psi^{\dagger}\left(i \partial_{t}+\frac{\nabla^{2}}{2 m}\right) \psi-\frac{C_{0}}{2}\left(\psi^{\dagger} \psi\right)^{2},
$$

with $m$ the (equal) mass of the two particles in the twobody system, and $\psi$ the matter field ${ }^{1}$. The two-body potential here is a three-dimensional delta function of strength $C_{0}$. That coefficient in front of that delta function is set by the two-body scattering length, $a$. Computation of the scattering amplitude, via the sum of diagrams depicted in Fig. 1, using, e.g. dimensional regularization with minimal subtraction [6] to calculate the loops ${ }^{2}$, leads to the series:

$$
t_{0}^{2 B}=\frac{4 \pi}{m}\left(a-i a^{2} k-a^{3} k^{2}+\ldots\right) .
$$

Here $k=\sqrt{m E}$ is the momentum of the two colliding particles in the center-of-mass frame.

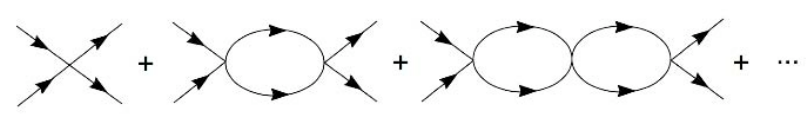

Fig. 1. The sum of diagrams needed to compute the leading-order two-body amplitude $t_{0}^{2 B}$. The vertices represent the $C_{0}$ contained in the leading-order $\mathcal{L}(1)$.

In Eq. (2) we see that quantum corrections to the treelevel amplitude are important as soon as $k \sim 1 / a$, and,

\footnotetext{
1 For particles with spin there are, in fact, two independent operators that can be written down. This is related to the presence of both a total-spin zero and a total-spin one channel. Since we will move back and forth between discussions of bosons and fermions we will obscure this technical detail in what follows, and assume that there is only a single channel for low-energy scattering, as is the case in bosonic systems.

2 Other schemes yield a more "natural" value for $C_{0}$, but the same physical amplitude.
}

once that regime is reached, all terms in the series are of the same order. Summation of the series produces:

$$
t_{0}^{2 B}=\frac{4 \pi a}{M} \frac{1}{1+i a k}
$$

an amplitude that will be valid for $k a \sim 1$, but $k \ell \ll 1$. Note that this amplitude predicts a pole at $k=i / a$, hence there is a two-body bound state with binding energy $B=1 / m a^{2}$ if $a>0$, and a low-energy pole but no bound state if $a<0$ (as occurs, e.g. in the ${ }^{1} \mathrm{~S}_{0}$ channel of $\mathrm{NN}$ scattering).

Eq. (3) is the "universal" result for $t^{2 B}$. It gives the amplitude if only the scattering length is known. To obtain predictions "beyond universality" we need to incorporate the effects of the finite range of the two-body potential in $t$. Sixty years ago, Bethe showed that $t^{2 B}(E)$ could be written in the form [7]:

$$
\begin{aligned}
t^{2 B}(E) & =-\frac{4 \pi}{m} \frac{1}{k \cot \delta(k)-i k} \\
k \cot \delta(k) & =-\frac{1}{a}+\frac{1}{2} r k^{2}+O\left(\ell^{3} k^{4}\right),
\end{aligned}
$$

with $k=\sqrt{m E}$ once again. The latter equation is known as the effective-range expansion, and is valid for $k \ell<1$. (More precisely, it is valid with a radius of convergence defined by the nearest singularity of the two-body potential.) In most quantum scattering situations $|r| \sim \ell$, and here we simply assume $|r| \ll|a|$. Then, provided that $k \sim 1 / a$, we may write $t$ as:

$$
t_{0}^{2 B}(E)+t_{1}^{2 B}(E)=\frac{4 \pi a}{m} \frac{1}{1+i a k}\left[1+\frac{1}{2} \frac{r k^{2}}{1+i a k}+\ldots\right]
$$

where the second term in square brackets is the next-toleading order (NLO) correction to the leading-order (LO) result. Note that Eqs. (4) and (5) only provide a more accurate representation of $t^{2 B}(E)$ than $t_{0}^{2 B}(E)+t_{1}^{2 B}(E)$ if $|r|$ is somehow appreciably larger than $\ell$.

An effective field theory (EFT) that reproduces the amplitude (6) order-by-order in an expansion in the ratio $r / a \sim$ $r k$ was set up in Refs. [8-12]. The leading-order Lagrangian in this EFT is Eq. (1). At sub-leading orders, interactions with more derivatives are inserted. For instance, insertions of:

$$
\mathcal{L}^{(2)}=\frac{C_{2}}{8}\left(\left(\psi^{\dagger} \psi\right)\left(\psi^{\dagger} \stackrel{\leftrightarrow}{\nabla}^{2} \psi\right)+\text { h.c. }\right),
$$

between the LO amplitude (3) in perturbation theory produce the second term in Eq. (6) $[8,9]$. This EFT is the "pionless EFT" in few-nucleon physics, but in this paper it will be used more generally than that, so hereafter we refer to it as "large-scattering-length EFT". This theory provides a way to systematically compute the effects of $r / a$ corrections to the universal predictions obtained from Eq. (1).

When $r / a$ and $r^{2} / a^{2}$ corrections are calculated in this EFT it results in a good description of NN S-wave phase shifts for momenta $k \sim 1 / a \ll 1 / \ell$. Numerically, it works a little better than one might expect given that the value of $\ell \sim 1 / m_{\pi}$ in the NN system, and failure is not seen until about $150 \mathrm{MeV}$-see Fig. 2. But this phenomenological 
$19^{\text {th }}$ International IUPAP Conference on Few-Body Problems in Physics

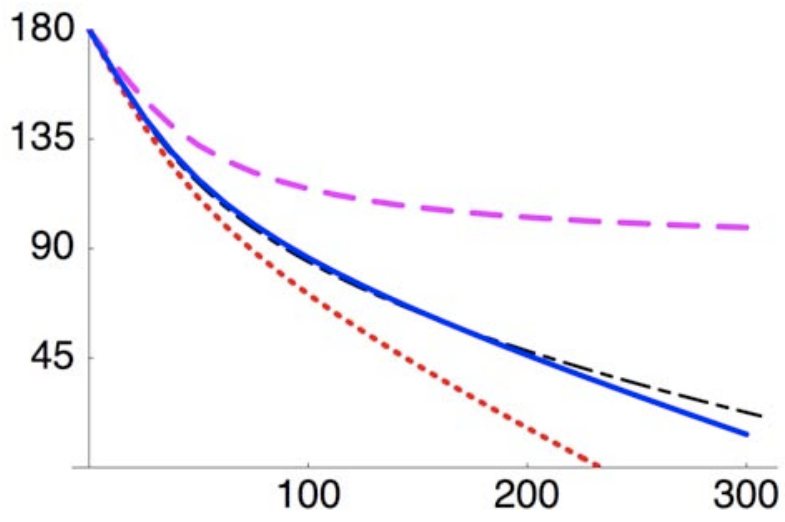

Fig. 2. NN scattering phases in the ${ }^{3} S_{1}$ channel (in degrees) versus center-of-mass momentum $k$ (in $\mathrm{MeV}$ ). Results are shown at LO (dashed line), NLO (dotted line), NNLO (solid line). The dot-dashed line represents the result of the Nijmegen phase-shift analysis [13]. Figure taken from Ref. [14].

success is really only proof that, as claimed, a re-expansion of the effective-range expansion is a useful tool.

To see the predictive power of the large-scattering-length EFT in the NN system one must examine electromagnetic (or, more generally, electroweak) reactions. Since we have an EFT, this can be straightforwardly done by adding to the Lagrangians (1), (7) etc. the terms required by minimal substitution, together with terms which contain operators that are gauge-invariant by themselves (e.g. nucleon magnetic moment operators). Diagrams for a given electromagnetic process can then be calculated, and the order of each one computed using the scaling $k \sim 1 / a$.

A quite successful example of this is the deuteron electromagnetic polarizability. This observable, denoted by $\alpha_{E 0}$, measures the extent to which the nucleus will develop an induced dipole moment in an applied electric field. $\alpha_{E 0}$ can be computed to four orders in the $r / a$ expansion, yielding to an accuracy of $1 \%$, if input solely from NN scattering data is employed [15]. For this purpose we use an alternative, slightly more efficient expansion of the $\mathrm{NN}^{3} \mathrm{~S}_{1}$ amplitude. That expansion is made around the deuteron pole by writing $k \cot \delta(k)=-\gamma+\rho\left(\gamma^{2}+k^{2}\right)+\ldots$ and expanding in powers of $\rho$. At leading order $\gamma=1 / a$ and $\rho=r$. But, the higher-order terms are different when this expansion is employed, as compared to what was written in Eq. (6). Numerically $\rho=1.7 \mathrm{fm}$ and $\gamma=0.23 \mathrm{fm}^{-1}$, so $\gamma \rho$ is the small parameter in this expansion, as seen explicitly in the result for $\alpha_{E 0}[16]$ :

$$
\alpha_{E 0}=\frac{\alpha_{e m} m}{32 \gamma^{4}}\left(1+\rho \gamma+\rho^{2} \gamma^{2}+\frac{2 \gamma^{2}}{3 m^{2}}+\ldots\right),
$$

with $\alpha_{e m}=e^{2} /(4 \pi \hbar c)$.

In the $\mathrm{NN}$ sector it is in electromagnetic reactions (see, in particular, Refs. $[15,16])$ that the physics of universality can be in probed in an unambiguous way. The fact that the leading-order prediction for $\alpha_{E 0}$ (and several other quantities too) depends only on $\gamma \sim 1 / a$ is a direct manifestation of universality, and suggests a universal result for the polarizability of any two-body bound state with a small binding energy. But the post-universal corrections to the deuteron polarizability are of order $1 / 3$, and so to truly check that the deuteron belongs to this set of universal bound states the inclusion of $\rho \gamma$ corrections is mandatory. By the time the NNLO corrections written in Eq. (8) are included, this is quite an accurate description.

A prominent example of this kind of success is the calculation of the reaction $\gamma \mathrm{d} \rightarrow \mathrm{np}$, relevant for Big-Bang Nucleosynthesis, to $1 \%$ accuracy [17]. That calculation was carried out to next-to-next-to-next-to-leading order $\left(\mathrm{N}^{3} \mathrm{LO}\right)$ in the large-scattering-length EFT. From this calculation it is very clear that $\ell /|a|$ corrections are crucial to getting an accurate description of the energy dependence of the deuteron photodisintegration cross section in the photon energy range from threshold up to $10 \mathrm{MeV}$. But, once these corrections are accounted for, and both the E1 and M1 transitions considered, the predictions of the EFT were consistent with all extant data in that energy range at the level of accuracy expected given that the first omitted correction is of order $(r / a)^{4}$.

\section{The three-body system: as a function of energy}

We now turn our attention to the three-body system. An extensive review of the leading-order predictions of the largescattering-length EFT for three-body systems can be found in Ref. [1], and these predictions were also discussed by Braaten in his contribution to this conference [2]. Here we summarize the LO predictions we need for our discussion of $\ell /|a|$ corrections, and then move on to explain how those corrections are computed.

\subsection{Leading order in the large-scattering-length EFT}

In Ref. [18] Bedaque, Hammer, and van Kolck considered the case of three bosons interacting via the Lagrangian (1). They calculated the amplitude for one of the bosons scattering from a bound state of the other two. In atomic physics this is the "atom-dimer scattering amplitude". In nuclear physics it is the "neutron-deuteron scattering amplitude". Hereafter the S-wave projected amplitude for this process, with scattering from relative-momentum state $k$ into relative-momentum state $p$, at energy $E$, will be denoted by $t_{0}^{3 B}$.

Ref. [18] showed that the Lagrangian (1) was incomplete, because the integral equation obtained from it that describes atom-dimer scattering yields results that are unstable with respect to the cutoff in the integral. I.e., those results depend on model assumptions about short-distance physics. To remedy this Bedaque et al. proposed an augmented LO Lagrangian, which is:

$$
\mathcal{L}=\psi^{\dagger}\left(i \partial_{t}+\frac{\vec{\nabla}^{2}}{2 m}\right) \psi-\frac{C_{0}}{2}\left(\psi^{\dagger} \psi\right)^{2}-\frac{H_{0}}{6 \Lambda^{2}}\left(\psi^{\dagger} \psi\right)^{3} .
$$


This yields the equation for the atom-dimer scattering amplitde:

$$
\begin{aligned}
& t_{0}^{3 B}(p, k ; E)= \\
& \frac{1}{p k}\left[\ln \frac{p^{2}+p k+k^{2}-m E-i \epsilon}{p^{2}-p k+k^{2}-m E-i \epsilon}+\frac{2 H_{0}(\Lambda) p k}{\Lambda^{2}}\right] \\
& +\frac{2}{\pi} \int_{0}^{\Lambda} d q \frac{q}{p}\left[\ln \frac{p^{2}+p q+q^{2}-m E-i \epsilon}{p^{2}-p q+q^{2}-m E-i \epsilon}+\frac{2 H_{0}(\Lambda) p q}{\Lambda^{2}}\right] \\
& \times \frac{t_{0}^{3 B}(q, k ; E)}{-1 / a+\sqrt{3 q^{2} / 4-m E-i \epsilon}} .
\end{aligned}
$$

If $H_{0}=0$ then this is the equation first derived by Skornyakov and Ter-Martirosian more than fifty years ago. It describes the three-body system interacting via zero-range two-body forces [19]. As has been known for many years (see, e.g., Ref. [20]), this equation is not well behaved, with the final result for $t_{0}^{3 B}$ depending strongly on the value chosen for $\Lambda$. This is exactly the reason given in Ref. [18] for introducing a"three-body force" $H_{0}(\Lambda)$, which is itself a function of the cutoff. At a given value of $\Lambda, H_{0}(\Lambda)$ must be chosen to reproduce one three-body observable. Ref. [18] showed that this removes this sensitivity of the low-energy amplitude to the choice of $\Lambda$. Afnan and Phillips subsequently demonstrated that this result can also be obtained by making a subtraction on the original integral equation, in order to render its kernel better behaved [21].

In either case, the conclusion is that a piece of threebody information is needed in order to employ the largescattering-length EFT in the three-boson system. Not all three-body systems built out of interactions with the same two-body scattering length are the same-even in the $|a| \rightarrow$ $\infty$ limit.

A standard choice for this piece of three-body input is to fix $H_{0}$ so that the energy of one three-body bound state in the unitary $(|a| \rightarrow \infty)$ limit is reproduced. We follow Ref. [1] and denote this energy as:

$$
E_{*} \equiv \frac{\hbar^{2} \kappa_{*}^{2}}{m} .
$$

Once this observable is fixed, predictions can be made for all other three-body observables at arbitrary scattering length (provided $|a| \gg \ell, k \ll 1 / \ell$ ). In particular, we find that, in the unitary limit, the $n$th three-body bound-state has binding energy:

$$
E_{n}=\left(e^{-2 \pi / s_{0}}\right)^{n-n_{*}} \frac{\hbar^{2} \kappa_{*}^{2}}{m},
$$

with $s_{0}=1.00624 \ldots$, if the $n *$ th state has energy $E_{*}$. In this way all binding energies in the unitary limit are determined once the one piece of three-body data, $E_{*}$, is known.

This reprises results of Thomas and Efimov: the threebody system with short-range two-body interactions alone is unstable against collapse [22]. But, if a piece of threebody information is added to the calculation, then, stable predictions-notably the prediction of a geometric spectrum in the unitary limit-are obtained [23]. For the purposes of this paper a key consequence of these observations is that the physics of a three-body system built on interactions with large two-body scattering lengths depends primarily on two numbers, $a$ and $\kappa_{*}$. Three-body systems still display "universality", because the details of the twobody interaction remain sub-dominant at low momenta. But $\kappa_{*}$ must be determined before predictions for threebody observables that are based on the universality of twobody interactions can be made.

In particular, the presence of this input from the threebody system breaks the continuous scale invariance that would otherwise be present in observables in the $|a| \rightarrow \infty$ limit. However, since the three-body spectrum in the unitary limit is geometric, the universal predictions are invariant under a rescaling of $\kappa_{*}$ by a factor of $e^{\pi / s_{0}} \approx 22.7$. Invariance under rescalings of dimensionful quantities by appropriate powers of this number is then a feature of threebody systems built out of two-body interactions with large scattering lengths. Such invariance is a prediction of universality: one we shall return to in what follows.

This invariance under rescaling by factors of $e^{\pi / s_{0}}$ is reflected in the asymptotic form of the atom-dimer scattering amplitude. For $p|a|, p / k \gg 1$, the amplitude $t_{0}^{3 B}(q, k ; E)$ is:

$$
\begin{aligned}
& t_{0}^{3 B}(q, k ; E)=\mathcal{N}\left(\frac{k}{\Lambda}\right)\left\{\frac{1}{q} \sin \left[s_{0} \ln \left(\frac{q}{\kappa_{*}}\right)\right]\right. \\
& \left.+\frac{8 \gamma\left|C_{-1}\right|}{q^{2}} \sin \left[s_{0} \ln \left(\frac{q}{\kappa_{*}}\right)+\arg \left(C_{-1}\right)\right]+\ldots\right\} .
\end{aligned}
$$

This form holds for all $q$ such that $q / k \gg 1$ and $q|a| \gg 1$. The dominant term here is the first one, $1 / q \sin \left[s_{0} \ln \left(q / \kappa_{*}\right)\right]$. The log periodicity of this dominant piece is the manifestation of discrete scale invariance in this quantity. However, the overall normalization of $t_{0}$ is a function of the on-shell momentum, $k: \mathcal{N}(k / \Lambda)$. Meanwhile the sub-leading term in the large- $q$ limit involves a complex number $C_{-1}$ that can be calculated from the Mellin transform of the asymptotic form of the kernel of the integral equation in Eq. (10) [24].

\subsection{Perturbation theory at NLO}

The first attempt to calculate range corrections to the LO three-body amplitude $t_{0}^{3 B}$ was made in Ref. [25] by Hammer and Mehen. They inserted the $O(r)$ correction from Eq. (6) between the leading-order three-body amplitude, and so computed the effects of that piece of (6) on the three-body system in first-order perturbation theory. In our notation the insertion is encoded as a "dimer" propagator:

$$
\mathcal{D}_{1}\left(q_{0}, \mathbf{q}\right)=-\frac{4 \pi i}{m g^{2}} \frac{r_{s}}{2} \frac{1 / a+\sqrt{-m q_{0}+\mathbf{q}^{2} / 4-i \epsilon}}{-1 / a+\sqrt{-m q_{0}+\mathbf{q}^{2} / 4-i \epsilon}},
$$

In Ref. [25] the constant piece of this propagator was not included in the analysis. It will be taken into account here. Projecting on $S$-waves we get:

$$
\begin{aligned}
& t_{1}^{3 B}(k, k ; E)= \\
& \int^{\Lambda} \mathrm{d} q \frac{r q^{2}}{\pi} \frac{1 / a+\sqrt{3 q^{2} / 4-m E-i \epsilon}}{-1 / a++\sqrt{3 q^{2} / 4-m E-i \epsilon}} t_{0}^{3 B^{2}}(k, q) \\
& +\frac{2 H_{1}(\Lambda)}{\Lambda^{2}}\left(1+\frac{2}{\pi} \int^{\Lambda} \mathrm{d} q \frac{q^{2} t_{0}^{3 B}(k, q)}{-1 / a+\sqrt{3 q^{2} / 4-m E-i \epsilon}}\right)^{2} .
\end{aligned}
$$




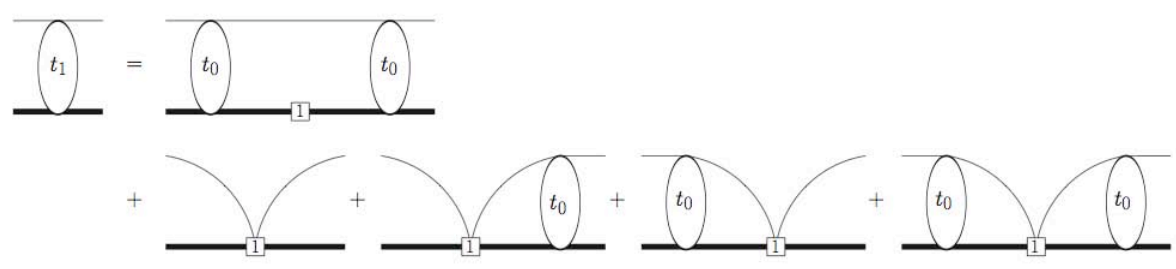

Fig. 3. The diagrams that need to be evaluated to yield the NLO amplitude in the three-body sector. $t_{1}$ represents the NLO 3B amplitude, and $t_{0}$ the LO amplitude. The vertex denoted by a "1" in the first diagram produces the $\sim r$ piece of the two-body propagator, $\mathcal{D}_{1}$, while the interactions labelled " 1 " in subsequent diagrams are proportional to $H_{1}$.

These terms are depicted in Fig. 3. The first of these displays a divergence that has to be canceled with the energyindependent three-body force $H_{1}(\Lambda)$ that appears in the second term-as was shown in Ref. [25]. The diagrams on the second line of Fig. 3 have to be evaluated for this purpose.

For fixed scattering length the new three-body force does not introduce an additional three-body parameter, since $H_{1}(\Lambda)$ is fixed by the renormalization condition that the NLO amplitude gives zero contribtion to the observable that was used for renormalization at leading order.

In Ref. [25] this approach was used to compute the NLO shift in the neutron-deuteron phase shifts in the $S=$ $1 / 2$ channel. The expression obtained there is slightly more complicated than Eq. (14), since one must consider NN scattering in both the ${ }^{1} \mathrm{~S}_{0}$ and ${ }^{3} \mathrm{~S}_{1}$ channels, but the principle is the same. The three-body observable used to fix $\kappa_{*}$ in Ref. [25] is the three-body scattering length in this $S=1 / 2$ channel, $a_{1 / 2}$ The results of this calculation are shown in Fig. 4. The NLO improvement is of the expected size, and brings the theory into better agreement with the data.

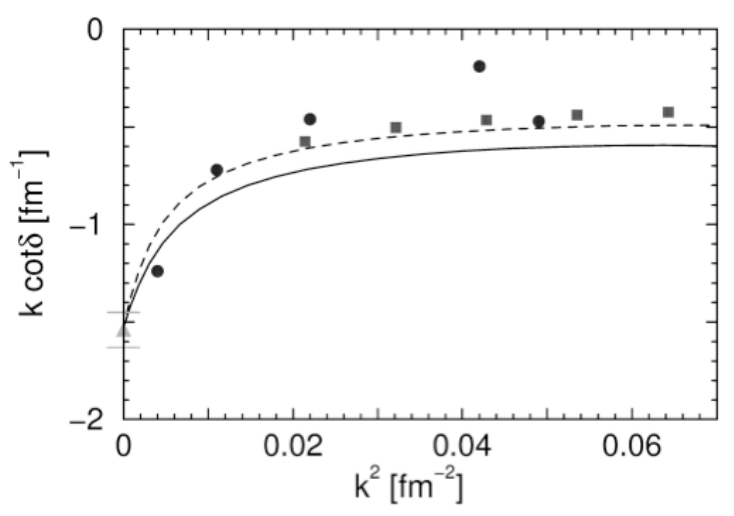

Fig. 4. Results for $S$-wave nd scattering in the spin- $1 / 2$ channel. The solid line is the LO result obtained in Ref. [18]. The dahsed line includes the $r / a$ corrections, calculated as described in this section. The squares are the result of a sophisticated potentialmodel calculation and the circles result from an older phase-shift analysis. Figure from Ref. [25], with permission.

\subsection{Some results at NNLO}

As yet this analysis of corrections to $t_{0}^{3 B}$ order-by-order in the $r / a$ expansion has not been extended to NNLO. Instead, in Ref. [24] the expansion of Eq. (6), carried out up to $O\left(r^{2}\right)$, was inserted as the NN amplitude in Eq. (10) in place of the leading-order two-body amplitude used there. The integral equation was then solved to obtain $t_{0}^{3 B}+t_{1}^{3 B}+$ $t_{2}^{3 B}+\ldots$. We note that this technique is only reliable for a range of cutoffs $\Lambda$ that obey $\Lambda \ell<1$. However, within this range Bedaque et al. performed an analysis, based on the asymptotic properties of the kernel, as well as numerical evidence, that demonstrated that another three-body input was needed at NNLO for renormalization. They used $a_{1 / 2}$, together with the triton binding energy, as these three-body inputs. WIth these two numbers, together with NN input on $a$ and $r$ in the ${ }^{1} \mathrm{~S}_{0}$ and ${ }^{3} \mathrm{~S}_{1}$ channels, in hand they predicted nd phase shifts for $k<m_{\pi}$ with considerable precision.

This calculation showed that in nuclear systems NNLO corrections can be $\sim 10 \%$. A recent NNLO calculation of the threshold cross section for the process nd $\rightarrow \mathrm{t} \gamma$, places similar emphasis on how important such effects beyond universality are for reproducing data [26]. Whereas the LO (or "universal") prediction for the threshold cross section is $\sigma=0.485 \mathrm{mb}$, the NNLO computation of Ref. [26] obtained $\sigma=0.503 \pm 0.003 \mathrm{mb}$. This is in excellent agreement with the experimental result $\sigma=0.508 \pm 0.015 \mathrm{mb}$ [27]. But the discrepancy would be several standard deviations if we stopped with the universal prediction. In this, capture reaction, effects "beyond universality" are seen too.

In contrast to these works, Refs. [28,29] showed that if the cutoff is taken to infinity in Eq. (10) with $t_{0}^{2 B}$ replaced by $t_{0}^{2 B}+t_{1}^{2 B}+t_{2}^{2 B}$, then a second three-body datum is not needed for renormalization. Only one three-body input (e.g. $\kappa_{*}$ ) is required up to this order. This suggests that in situations where $|r| \gg \ell$ the predictive power of the theory is greater than is implied by the analysis of Ref. [24].

\section{The three-body system: as a function of scattering length}

\subsection{Next-to-leading-order analysis in the large-scattering-length EFT}

In Ref. [30] this observation was used as the basis of a calculation of the scattering-length dependence of the boundstates in the three-body system in the presence of a finite 
effective range. As $a$ is varied in the two-body system the energies at which three-body bound states change occur. Away from the unitary limit we may write the binding energies as:

$$
E_{n}=\frac{\hbar^{2} \kappa_{n}^{2}}{m}\left[F\left(\frac{1}{\kappa_{n} a}\right)+\kappa_{*} r_{s} G_{n}\left(\frac{1}{\kappa_{*} a}\right)+\ldots\right],
$$

where $\kappa_{n}=\kappa_{*} e^{-(n-n *) \pi / s_{0}}$ and $F(0)=1$ by construction, so that this formula agrees with Eq. (12) in the unitary limit. As was discussed by Braaten at this meeting [2], the discrete scale invariance in the unitary limit persists to finite $a$ because states with different $n$ have binding energies related by factors of $e^{\pi / s_{0}} \approx 22.7$ if $a$ is rescaled by a factor of $e^{\pi / s_{0}}$. The function $F$ was computed by Efimov, and is parameterized in Ref. [1].

In Ref. [30] we showed rigorously that $G_{n}(0)=0$ for all $n$, i.e. there is no shift in any $\kappa_{n}$ in the unitary limit ${ }^{3}$. This is a consequence of the discrete scale invariance of the leading-order wave functions. We then extended the Efimov plot to NLO by solving Eq. (10) with $t$ up to first order (see Eq. (6)) as input. The results of this exercise are shown in Fig. 5. A contemporaneous study by Thøgerson et al. used a potential model to produce results that were in qualitative and quantitative agreement with ours [31].

One might think that the results of Ref. [30] would be reproduced by a strictly perturbative analysis of the NLO shift in the Efimov spectrum, and that is true in the limit $|r| \gg \ell$. However, in a perturbative analysis the second term in Eq. (14) generates divergences:

$$
\begin{aligned}
t_{1}^{3 B} & \sim r \int^{\Lambda} \mathrm{d} q q^{2} \frac{1 / a+\sqrt{3} q / 2}{-1 / a+\sqrt{3} q / 2} t_{0}^{3 B^{2}}(k, q) \\
& \sim r \Lambda+\frac{r}{a} \log (\Lambda a)+\ldots
\end{aligned}
$$

as $\Lambda \rightarrow \infty$. The linear divergence is renormalized away, by a piece to $H_{1}$ that $\sim r \Lambda$ and is independent of $a$. The binding energies of states in the unitary limit are hence unaffected by the NLO correction, as shown in Ref. [30]. But the presence of the log divergence proportional to $r / a$ means that $H_{1}$ must also have an $a$-dependent piece. Hence another three-body datum-one besides $\kappa_{*}$-is needed to predict the $a$-dependence of the spectrum of three-body states at NLO. Furthermore, that three-body datum cannot be determined from unitary limit observables, because it is only probed at $|a| \neq \infty$. One non-unitary-limit observable in the three-body system is needed in order to completely determine the NLO correction. Hence, Eq. (15) above is incomplete.

The existence of such an $a$-dependent three-body force can also be understood by considering the Lagrangian of the EFT with contact interactions alone. This Lagrangian should include all terms allowed by the symmetries of the system. In our problem there is no symmetry that precludes the presence of terms proportional to $1 / a$. Thus, beyond

\footnotetext{
3 This conclusion holds as long as $\kappa_{*}$ is the observable used for renormalization.
}

LO, the three-body term in Eq. (9) must be augmented to:

$$
\frac{H_{0}}{6 \Lambda^{2}}\left(\psi^{\dagger} \psi\right)^{3}+\frac{r}{a} \frac{\tilde{H}_{1,1}}{6 \Lambda^{2}}\left(\psi^{\dagger} \psi\right)^{3}+\left(\frac{r}{a}\right)^{2} \frac{\tilde{H}_{2,2}}{6 \Lambda^{2}}\left(\psi^{\dagger} \psi\right)^{3}+\ldots
$$

The presence of such $a$-dependent three-body interactions is analogous to the need to consider the pion-mass dependence of the short-range part of the $\mathrm{NN}$ interactions in chiral effective theories $[6,8,9]$. And as with the $m_{\pi^{-}}$ dependent NN forces of the chiral EFT, the $a$-dependent three-body forces in the large-scattering-length EFT are mandatory for correct renormalization.

If $\ell \ll|r|$, then the renormalized part of $H_{1,1}, H_{1,1}^{(r)}$ is small if evaluated at scale $1 / \ell$. This justifies the treatment of Ref. [30] in that limit, since the rest of the $1 / a$ dependence of observables is described by long-distance physics at this order. If $\ell \ll|r| \ll|a|$, Eq. (15) is valid once more, since the additional three-body datum needed at NLO has only a parameterically small impact on observables. But, if $|r| \sim \ell$, then the effects of $H_{1,1}$ must be considered.

\subsection{Application to data on recombination in cold atomic gases}

These effects can be discerned experimentally in the $a$ dependence of various features in recombination experiments with cold atoms. As described further in various presentations at this meeting, the use of Feshbach resonances allows us to take trapped atomic gases, and then vary atomatom scattering lengths over wide ranges by adjusting the magnetic field. Monitoring the atomic loss rate as a function of that magnetic field then provides information on the spectrum of three-body "Efimov" states, since we can thereby trace the rates of atomic recombination processes that drive these losses.

Three features in the recombination rate have received particular attention. First, the atom-dimer threshold can be observed through a resonance feature in recombination, and the scattering length at which this occurs is denoted by $a_{*}$. Second, there is a maximum in the recombination rate at the negative scattering length for which the threebody bound state has zero energy, as then recombination is enhanced because it can proceed through capture into this bound state. That particular scattering length is denoted by $a^{\prime}$ in what follows. Conversely, there is a minimum in the recombination rate at a positive scattering length, $a_{0}$, where $t^{3 B}=0$. This zero in the scattering amplitude is sometimes understood as being due to destructive interference of two different recombination-reaction pathways.

In the limit $r=0$ the EFT discussed above can be used to predict "universal relations" between these three different scattering lengths. We have [1]

$$
a_{0} / a^{\prime}=-4.88 e^{n \pi / s_{0}} ; \quad a^{\prime} / a_{*}=-0.9727 e^{n \pi / s_{0}} .
$$

These constitute LO predictions in the EFT for large scattering lengths. If one of the recombination features is given, it can be used to set $\kappa_{*}$, and the position of others can be predicted. 


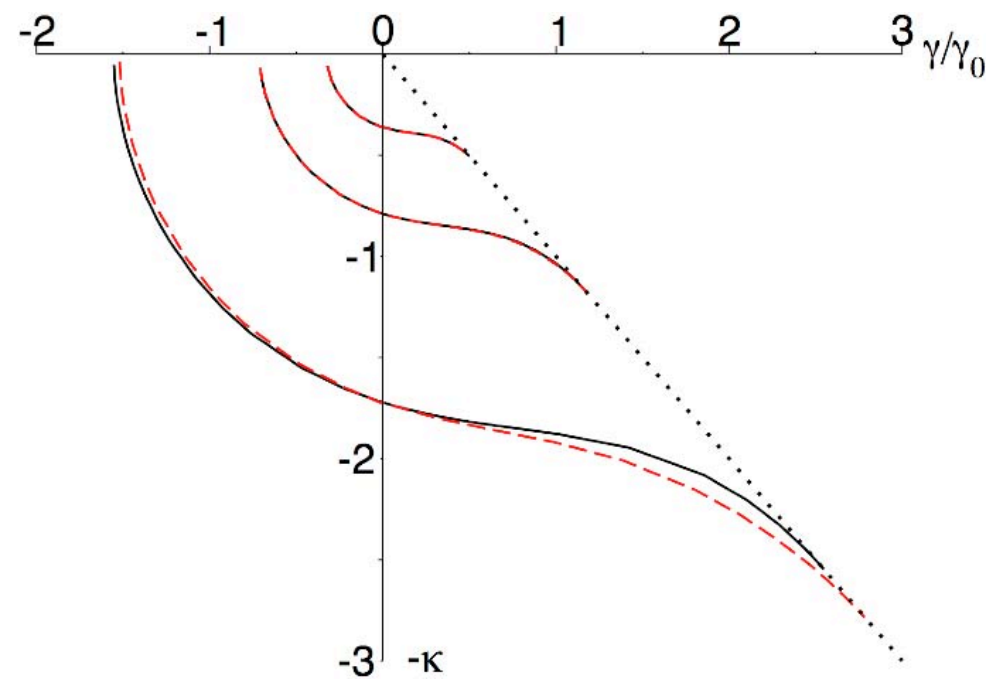

Fig. 5. The bound-state spectrum of the three-body system with short-range interactions. The solid lines denote the LO results, and the dashed lines give the NLO result for the case $r_{s}=0.01$ (in arbitrary units). Here the scale has been altered as per Ref. [1], with $\gamma=1 / a$ and $\kappa$ first rewritten as $\gamma=\gamma_{0} H \cos \zeta$ and $\mid \gamma=\gamma_{0} H \sin \zeta$ and then the quantities $H^{1 / 4} \cos \zeta$ and $-H^{1 / 4} \sin \zeta$ displayed here. The dotted line denotes the point at which three-body bound states become unstable against breakup to a two-body bound state and a free particle. Figure after Ref. [30].

The relationships (18) are borne out fairly well in experiments with Lithium-7 atoms [32], which yield:

$$
a^{\prime}=-264(11) a_{B} ; \quad a_{0} \approx 1160 a_{B},
$$

with $a_{B}$ the Bohr radius. However, the experiments of the Innsbruck group $[33,34]$ with Cesium atoms found values which do not obey (18) so well: $a_{0} \approx 210 a_{B}, a_{*} \approx 367 a_{B}$, and $a^{\prime} \approx-850 a_{B}$. While the LO prediction works quite nicely for the results (19), in the case of Ref. [33] it is not very well obeyed. The first equation in (18) would predict:

$$
a^{\prime}=-1025 a_{B}
$$

based on the measured value of $a_{0}$. But $a_{0}=210 a_{B}$ is not much larger than the van der Waal's scale in Cesium $\ell \sim 70 a_{B}$. Therefore, it seems important to discuss how NLO corrections in the theory with large scattering lengths modify predictions for recombination in Cesium.

In Fig. 6 we show a LO and NLO prediction for the recombination length $\rho_{3}$ for Cesium atoms. This is related to the recombination rate $\alpha$, by:

$$
\rho_{3}=\left(\frac{2 \sqrt{3} \alpha}{\hbar}\right)^{1 / 4} .
$$

The data shown is from Ref. [33]. The LO prediction is adjusted to give the value $a_{0}=210 a_{B}$ extracted in Ref. [33]. This corresponds to a value of $\kappa_{*}$ of $1.52 \times 10^{-3} \hbar a_{B}^{-1}$. At NLO we adjust $H_{0}$ to keep this same value of $\kappa_{*}$. But, without a non-zero $H_{1,1}$, the result for this observable is not cutoff independent. So, $H_{1,1}(\Lambda)$ is tuned to reproduce the value of $a^{\prime}$. NLO corrections go as $r / a$ in this renormalization scheme.

Three things are clear from Fig. 6. First, in the vicinity of the recombination minimum the NLO correction to the LO result is of order $100 \%$. Of course, minima are always hard to reproduce, because by definition the amplitude vanishes there, and so effects that are small elsewhere can make a big difference in the observable there. But, the NLO correction does not become a small perturbation to the LO result until $a \approx 300-400 a_{B}$. This leads us to our second point: data on $\rho_{3}$ at scattering lengths larger than that are quite well reproduced by the NLO result. Thus, the predictions of the large-scattering length EFT at values of $a$ which are large enough that the EFT can be trusted are in accord with the observation of $a^{\prime}=-850 a_{B}$ and the leading-order value of $\kappa_{*}$. We therefore conclude that NLO corrections may well account for the difference between these data and the universal prediction based on the measured $a_{0}$, Eq. (20). Third, noting the difference between the second minimum in the red curve and the first one in the blue curve we see that the LO prediction of the EFT for the position of the recombination minimum at $a \sim 3000 a_{B}$ is appreciably affected by higher-order corrections. The different recombination minima are no longer separated by a factor of $e^{\pi / s_{0}}$ in $a$ once NLO corrections are considered, because NLO corrections to $\rho_{3}$ break discrete scale invariance. Indeed, if we had chosen a renormalization scheme where $a^{\prime}$ and $a_{*}$ (say) were both exactly reproduced at NLO there would be an NLO shift even in the limit $|a| \rightarrow \infty$. In such a scheme the recombination minimum at $a^{\prime} \sim 80,000 a_{B}$ is also shifted by NLO corrections.

The optimal renormalization scheme at NLO is the one that minimizes still higher-order corrections to observables. The scheme that satisfies that criterion is yet to be determined. At NLO one can work in different schemes and produce predictions for the $\rho_{3}$ vs $a$ curve. The differences between the results in different schemes then give an estimate of the uncertainty due to NNLO effects.

In addition, care must be exercised because in the vicinity of a zero in the scattering length, $r$ varies quite dramat- 


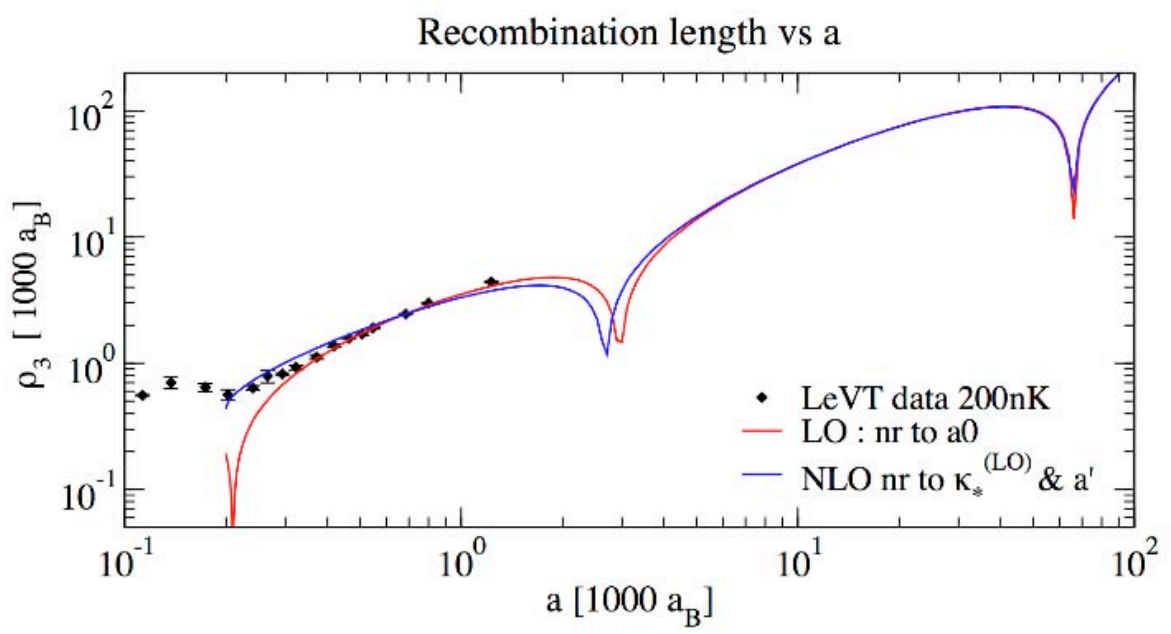

Fig. 6. Predictions at LO and NLO for the "recombination length" $\rho_{3}$ compared with data from Ref. [33]. The red line is the leading-order result, in which $a_{0}$ is taken as the three-body datum, and the blue curve is the NLO result, in which we have renormalized to the same $\kappa_{*}$ as at LO, and the value of $a^{\prime}$ measured in Ref. [33]. The value of $r$ was chosen to be $r=100 a_{B}$.

ically with magnetic field, with $r \rightarrow-\infty$ when $a=0$. The data taken in Ref. [33], and displayed in Fig. 6, were taken in the vicinity of such a zero, and so, for $a \approx 100-200 a_{B}$ the value of $r$ can be much larger than $100 a_{B}$, and of opposite sign. A full treatment of these data therefore requires us to include the impact of an effective range that varies with magnetic field on $\rho_{3}$. The fact that data in the vicinity of the recombination minimum are not well described in Fig. 6 is therefore not of concern, given that such effects were not accounted for there.

The curves shown in Fig. 6 also do not contain corrections for the effects of finite temperature or deep dimers. Effects due to the latter will, for instance, fill in the recombination minima and make the predictions much smoother as a function of $a$. One way to look at the theory prediction without having to worry about this issue is to ask how linear-in- $r$ effects modify the universal relations (18). The answer to this question depends somewhat on renormalization scheme, but to derive these relations here we work in a renormalization scheme where $a_{0}$ is fixed at LO, and at NLO we keep the same $a_{0}$ and $\kappa_{*}$.

The pattern of NLO corrections then depends on the value of $n$ chosen in Eq. (18), because range corrections break discrete scale invariance, and so they affect the different states in the Efimov spectrum differently. For $n=-1$ we find, for instance:

$$
a^{\prime}=-0.215 a_{0}+1.18 r+O\left(r^{2} / a_{0}\right) .
$$

It is not clear that we can apply such an equation-even if we choose one for a different $n$-to relate the $a_{0}$ and $a^{\prime}$ measured in Ref. [33], since those two quantities are connected through a zero of the scattering length. The correct value of $r$ to insert is thus not clear. (It is also not clear that we can use the same three-body parameter $\kappa_{*}$ on both sides of the point $a=0$.) We can, however, apply Eq. (22) to the findings of Ref. [32]. There, recombination features were measured on either side of a Feshbach resonance where $|a| \rightarrow \infty$ and $r=-15 a_{B}$ [35]. For the $a_{0}$ given in Eq. (19), and using this effective range, we have:

$$
a^{\prime}=(-249-17+\ldots) a_{B} .
$$

This shows that the range corrections in the Lithium-7 system help to improve the agreement between theory and the experimentally measured $a^{\prime}$ quoted above.

The effect of range corrections in these data is not large, because the $|r|$ is appreciably smaller than $\ell$ in the vicinity of the broad Feshbach resonance used by Gross et al. for this experiment [36]. But, with accurate determinations of $a^{\prime}$ and $a_{0}$, the impact of the term proportional to $r$ in Eq. (22) will become discernible. A proper treatment of those terms necessitates the inclusion of the piece of shortdistance, three-body, physics encoded in the parameter $H_{1,1}$.

\section{Some words on the four-body system}

We now move on to consider effects beyond universality in the four-body system. As discussed elsewhere in these proceedings $[2,37]$, the prediction of universality-or, equivalently, the leading-order prediction in the large-scatteringlength EFT-is that near $|a|=\infty$ there will be two fourbody bound states for each three-body bound state. Moreover, the binding energies of these states will be related to that of the three-body state, $B$, by $[39,40]$ :

$$
B_{4,0} \approx 5.0 B ; B_{4,1} \approx 1.01 B \text {. }
$$

This prediction has recently been confirmed in four-body recombination data [38].

In the four-nucleon system it is well established that there is a correlation-known from a phenomenological point of view for many years and dubbed the "Tjon line"like the first one in Eq. (24), i.e. between the energy of the three-nucleon and four-nucleon bound states. In Ref. [41] 


\section{$19^{\text {th }}$ International IUPAP Conference on Few-Body Problems in Physics}

Platter, Hammer, and Meißner showed that this correlation was a prediction of the large-scattering-length EFT at leading order. It can be used to predict the binding energy of the Helium-4 nucleus, if the binding energy of the triton is given. With only the binding energy of the $A=2$ and $A=3$ nuclides and the ${ }^{1} \mathrm{~S}_{0}$ scattering length as input Platter et al. predict $B_{4,0}=27 \pm 2 \mathrm{MeV}$ for $\mathrm{A}=4$, in remarkably good agreement with experiment.

The shallower of the two four-nucleon bound states predicted by universality will be rendered unbound by the Coulomb interaction. Thus it can only be probed in scattering experiments or through electromagnetic observables. Future investigations that combine theory and experiment in order to check the extent to which the prediction (24) is obeyed by the strong interactions in the $A=4$ system could be extremely fruitful.

One aspect of such investigations will be a proper treatment of $r / a$ corrections. Recently, Kirscher et al. made the first examination of $r / a$ corrections to four-nucleon observables in the large-scattering-length EFT. They find that these corrections narrow the correlation band between $B_{4,0}$ and $B_{3}$ and produce similarly tight correlation bands between the triton binding energy and the Helium-3-neutron scattering length (see Fig. 7). Measurements of the latter quantity can serve to identify the presence of $r / a$ corrections to the "universal" correlations between three- and four-body system observables in systems with large scattering length.

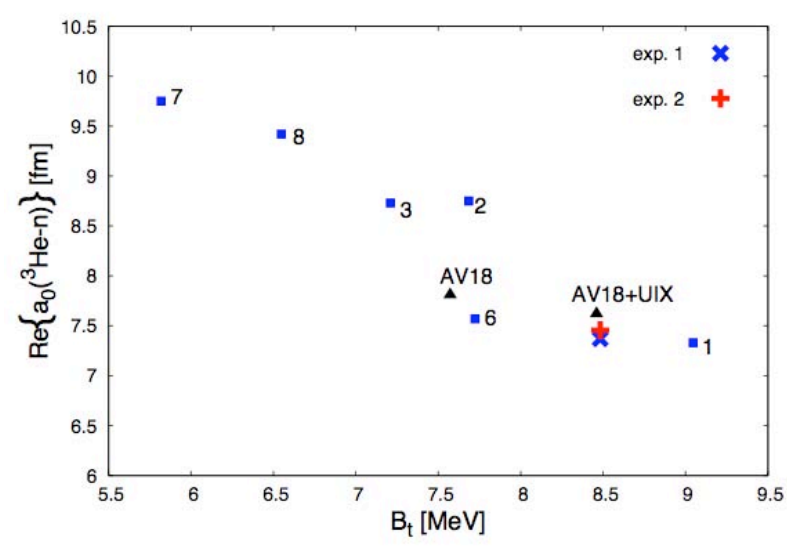

Fig. 7. Predictions at NLO for the correlation between the triton binding energy and the real part of the neutron-Helium-3 scattering length. The different numbered points correspond to different implementations of the NLO EFT calculation, while the points labelled AV18 and AV18 + UIX indicate the results of sophisticated potential-model calculations with and without three-body forces, respectively. The plus and the cross indicate the results of two recent experiments. For details see Ref. [42].

\section{Conclusion}

Universality links gases of cold atoms, few-nucleon systems, halo nuclei, and hadronic molecules. These systems, although their energy scales are vastly different, have in common that they all involve large two-body scattering lengths. They differ from one another in the pattern of their $r / a$ corrections: the way they manifest effects "beyond universality".

In few-nucleon systems (and in halo nuclei too, see Refs. $[43,44])$ understanding such corrections is mandatory if an accurate description of data is to be obtained. In contrast, the gross features of atomic losses near Feshbach resonances due to three- and four-body recombination are already surprisingly well explained by the predictions of universality.

These predictions constitute the LO results in the largescattering-length EFT developed in Refs. [8-12,18]. But, range corrections are key to systematic control of effects in different atomic systems. While such corrections are small for the data obtained in Ref. [32], they can be expected to be sizeable in the vicinity of the recombination minimum observed in Ref. [33]. A key finding reported here is that an extra three-body datum is needed to fully constrain the scattering-length dependence of these range corrections.

A study of $r / a$ corrections in the context of four-body recombination in atomic systems would help identify how far the exciting observations reported at this meeting [37, 38 ] are a result of "universality" in systems with large scattering length, and how far they are specific to the Cesium gas used for those experiments. The formalism to carry out such calculations "beyond universality" already exists. It is a straightforward extension of the techniques already implemented in Refs. [41,42], and would be a useful application of the next-to-leading-order two-body and three-body interactions described in this talk.

This contribution provides only a short and selective introduction to the way universality and its corrections drive physics in few-nucleon systems and cold atomic gases. A more extensive discussion along these lines can be found in the recent review [45].

\section{Acknowledgments}

It is a pleasure to acknowledge my collaborators in the work that constitutes the bulk of the new results presented here: Chen Ji and Lucas Platter. I am also grateful to Lucas for many conversations and much education on recombination in cold atomic gases. I also thank Eric Braaten, David Canham, Harald Grießhammer, and Hans-Werner Hammer for discussions on the topics I have presented here. This work is supported by the US Department of Energy DEFG02-93ER-40756 and by the Mercator programme of the Deutsche Forschungsgemeinschaft.

\section{References}

1. E. Braaten and H.-W. Hammer, Phys. Rept. 428 (2006) 259.

2. E. Braaten, these proceedings.

3. T. Mehen, these proceedings.

4. S. Weinberg, Phys. Lett. B 251 1990, 288. 
5. S. Weinberg, Nucl. Phys. B 363,(1991) 3.

6. D. B. Kaplan, M. J. Savage and M. B. Wise, Nucl. Phys. B 478 (1996) 629.

7. H. A. Bethe, Phys. Rev. 76 (1949) 38.

8. D. B. Kaplan, M. J. Savage and M. B. Wise, Phys. Lett. B 424 (1998) 390.

9. D. B. Kaplan, M. J. Savage and M. B. Wise, Nucl. Phys. B 534 (1998) 329.

10. U. van Kolck, Nucl. Phys. A 645 (1999) 273;

11. J. Gegelia, Phys. Lett. B 429 (1998) 227.

12. M. C. Birse, J. A. McGovern and K. G. Richardson, Phys. Lett. B 464 (1999) 169.

13. V. G. J. Stoks, R. A. M. Klomp, M. C. M. Rentmeester and J. J. de Swart, Phys. Rev. C 48 (1993) 792.

14. S. R. Beane, P. F. Bedaque, W. C. Haxton, D. R. Phillips and M. J. Savage, "From hadrons to nuclei: Crossing the border," in the Borris Ioffe Festschrift, "At the frontier of particle physics: handbook of QCD”, M. Shifman (ed.), nucl-th/0008064.

15. D. R. Phillips, G. Rupak and M. J. Savage, Phys. Lett. B 473 (2000) 209.

16. J. W. Chen, G. Rupak and M. J. Savage, Nucl. Phys. A 653 (1999) 386.

17. G. Rupak, Nucl. Phys. A 678 (2000) 405.

18. P. F. Bedaque, H. W. Hammer and U. van Kolck, Phys. Rev. Lett. 82 (1999) 463; Nucl. Phys. A 646 (1999) 444.

19. G.V. Skorniakov and K.A. Ter-Martirosian, Sov. Phys. JETP 4, 648 (1957) [J. Exptl. Theoret. Phys. (U.S.S.R.) 31, 775 (1956)].

20. G.S. Danilov, Sov. Phys. JETP 13, 349 (1961).

21. I. R. Afnan and D. R. Phillips, Phys. Rev. C 69 (2004) 034010.

22. L. H. Thomas, Phys. Rev. 47 (1935) 903

23. V. Efimov, Phys. Lett. B 33 (1970) 563.

24. P. F. Bedaque, G. Rupak, H. W. Grießhammer and H.W. Hammer, Nucl. Phys. A 714 (2003) 589.

25. H.-W. Hammer and T. Mehen, Phys. Lett. B 516 (2001) 353.

26. H. Sadeghi, S. Bayegan, H. W. Grießahmmer, Phys. Lett. B 643 (2006) 263.

27. E. T. Jurney, P. J. Bendt and J. C. Browne, Phys. Rev. C 25 (1982) 2810.

28. L. Platter and D. R. Phillips, Few Body Syst. 40 (2006) 35.

29. L. Platter, Phys. Rev. C 74 (2006) 037001.

30. L. Platter, C. Ji and D. R. Phillips, Phys. Rev. A 79 (2009) 022702.

31. M. Thøgersen, D. V. Fedorov and A. S. Jensen, Phys. Rev. A 78, 020501 (2008).

32. N. Gross, Z. Shotan, S. Kokkelmans, L. Khaykovich, Phys. Rev. Lett. 103 (2009) 163202.

33. M. Kraemer et al., Nature 440 (2006) 315.

34. S. Knoop et al., Nature Physics 5 (2009) 227.

35. S. Kokkelmans, private communication.

36. E. Braaten, D. Kang and L. Platter, Phys. Rev. A 78 (2008) 053606.

37. F. Ferlaino, these proceedings.

38. F. Ferlaino et al., Phys. Rev. Lett. 102 (2009) 140401.
39. H.-W. Hammer and L. Platter, Eur. Phys. J. A 32 (2007) 113.

40. J. von Stecher, J. d'Incao, C. Greene, Nature Physics 5 (2009) 417.

41. L. Platter, H.-W. Hammer and U. G. Meißner, Phys. Lett. B 607 (2005) 254.

42. J. Kirscher, H. W. Grießhammer, D. Shukla and H. M. Hofmann, arXiv:0903.5538 [nucl-th].

43. D. L. Canham and H.-W. Hammer, arXiv:0911.3238 [nucl-th].

44. D. R. Phillips and H.-W. Hammer, these proceedings.

45. L. Platter, Few Body Syst. 46, 139 (2009). 